\title{
A Uniform Resource Name (URN) Namespace for the Motion Picture Experts Group (MPEG)
}

Status of this Memo

This memo provides information for the Internet community. It does not specify an Internet standard of any kind. Distribution of this memo is unlimited.

Copyright Notice

Copyright (C) The Internet Society (2003). All Rights Reserved.

Abstract

This document describes a Uniform Resource Name (URN) namespace for the Motion Picture Experts Group (MPEG) for naming persistent resources as part of the MPEG standards. Example resources include technical documents and specifications, extensible Markup Language (XML) Schemas, classification schemes, XML Document Type Definitions (DTDs), namespaces, style sheets, media assets, and other types of resources produced or managed by MPEG.

\section{Introduction}

MPEG is a working group of the International Organization for Standardization (ISO) and International Engineering Consortium (IEC) in charge of the development of standards for coded representation of digital audio and video. MPEG has produced MPEG-1, the standard on which such products as Video CD and MP3 are based, MPEG-2, the standard on which such products as Digital Television set top boxes and DVD are based, MPEG-4, the standard for multimedia for the fixed and mobile web, and MPEG-7, "Multimedia Content Description Interface", the standard for description and search of audio and visual content. Work on MPEG-21, "Multimedia Framework" is currently underway.

MPEG would like to assign unique, permanent, location-independent names based on URNs for some resources it produces or manages.

This namespace specification is for a formal namespace. 


\section{Specification Template}

Namespace ID:

"mpeg"

Registration Information:

Version: 1

Date: 2001-11-20

Declared registrant of the namespace:

Name: John R. Smith

Title: Chair, MPEG MDS Subgroup

Affiliation: IBM T. J. Watson Research Center

Address: $\quad 30$ Saw Mill River Road

Phone: $\quad+1$ (914) $784-7320$

Email: jrsmithewatson.ibm.com

Declaration of structure:

URNs assigned by MPEG will have the following hierarchical structure based on the organizational structure of the MPEG standards:

urn:mpeg: $\{$ standard name $\}:\{$ assigned US-ASCII string

where "\{standard name\}" is a US-ASCII string that conforms to URN Syntax requirements ([RFC2141]) and corresponds to the name of an MPEG standard (such as "mpeg1", "mpeg2", "mpeg4", "mpeg7",

"mpeg21", and

"\{assigned US-ASCII string\}" is a US-ASCII string that conforms to URN Syntax requirements ([RFC2141]).

The individual URNs shall be assigned by MPEG through the process of development of MPEG standards.

Relevant ancillary documentation:

None

Identifier uniqueness considerations:

MPEG shall establish unique identifiers as appropriate. 
Uniqueness is guaranteed as long as the assigned string is never reassigned for a given standard name and that the standard name is never reassigned.

Identifier persistence considerations:

MPEG is committed to maintaining the accessibility and persistence of all resources that are officially assigned URNs by the organization.

Persistence of identifiers is dependent upon suitable delegation of resolution at the level of "standard name"(s), and persistence of standard name assignment.

Process of identifier assignment:

Assignment is limited to the owner and those authorities that are specifically designated by the owner. MPEG may designate portions of its namespace for assignment by other parties.

Process of identifier resolution:

The owner will develop and maintain "URN catalogs" that map all assigned URNs to Uniform Resource Locators (URLs) specifically to enable Web-based resolution of named resources. In the future an interactive online resolution system may be developed to automate this process.

The owner will authorize additional resolution services as appropriate.

Rules for Lexical Equivalence:

The "standard name" is case-insensitive. Thus, the portion of the URN :

urn:mpeg: $\{$ standard name $\}$ :

is case-insensitive for matches. The remainder of the identifier must be considered case-sensitive.

Conformance with URN Syntax:

No special considerations.

Validation mechanism:

None specified. The owner will develop and maintain URN catalogs. 
The presence of a URN in a catalog indicates that it is valid.

Scope :

Global

3. Examples

The following examples are not guaranteed to be real. They are presented for pedagogical reasons only.

urn :mpeg: mpeg $7:$ schema : 2001

urn:mpeg:mpeg $7:$ cs:VideoDomainCs: 2001

urn:mpeg:mpeg $7:$ cs: GenreCs: 2001

urn:mpeg : mpeg $7: \mathrm{cs}:$ ContentCS: 2001

4. Namespace Considerations

URN assignment procedures:

The individual URNs shall be assigned through the process of development of MPEG standards by the Moving Picture Experts Group (MPEG), which is a working group of ISO/IEC.

URN resolution/delegation:

The resolution and delegation shall be determined through the process of development of MPEG standards by the Moving Picture Experts Group (MPEG). MPEG has developed requests for registration authority for MPEG-7 classification schemes and MPEG-21 identification systems. These registration authorities shall be responsible for corresponding parts of MPEG namespace.

Type of resources to be identified:

Types of resources to be identified include XML schema definition files, classification schemes, identification systems.

Type of services to be supported:

Types of services supported include controlled term lookup in classification schemes, resolution of ids in identification systems. 
5. Community Considerations

Open assignment and use of identifiers within the namespace:

With on-going development of MPEG standards, MPEG shall establish requirements for assignment and use of identifiers within MPEG namespace. MPEG has already established requirements for assignment and use of MPEG-7 classification schemes under MPEG namespace. A registration authority shall have the responsibility for processing third party requests for registering classification schemes.

Open operation of resolution servers for the namespace (server):

With on-going development of MPEG standards, MPEG shall establish requirements and seek candidates for operating resolution servers as appropriate.

Creation of software that can meaningfully resolve and access services for the namespace (client):

With on-going development of MPEG standards, MPEG shall develop reference software implementations of its standards.

6. Security Considerations

There are no additional security considerations other than those normally associated with the use and resolution of URNs in general.

7. IANA Considerations

The IANA has registered formal URN namespace 12, to MPEG within the IANA registry of URN NIDs.

8. Normative References

[RFC2141] Moats, R., "URN Syntax", RFC 2141, May 1997.

9. Author's Address

John R. Smith

IBM T. J. Watson Research Center

30 Saw Mill River Road

Hawthorne, NY 10532 USA

Phone: 1 (914) 784-7320

EMail: jrsmith@watson.ibm.com 
10. Full Copyright statement

Copyright (C) The Internet Society (2003). All Rights Reserved.

This document and translations of it may be copied and furnished to others, and derivative works that comment on or otherwise explain it or assist in its implementation may be prepared, copied, published and distributed, in whole or in part, without restriction of any kind, provided that the above copyright notice and this paragraph are included on all such copies and derivative works. However, this document itself may not be modified in any way, such as by removing the copyright notice or references to the Internet society or other Internet organizations, except as needed for the purpose of developing Internet standards in which case the procedures for copyrights defined in the Internet Standards process must be followed, or as required to translate it into languages other than English.

The limited permissions granted above are perpetual and will not be revoked by the Internet society or its successors or assignees.

This document and the information contained herein is provided on an "AS IS" basis and THE INTERNET SOCIETY AND THE INTERNET ENGINEERING TASK FORCE DISCLAIMS ALL WARRANTIES, EXPRESS OR IMPLIED, INCLUDING BUT NOT LIMITED TO ANY WARRANTY THAT THE USE OF THE INFORMATION HEREIN WILL NOT INFRINGE ANY RIGHTS OR ANY IMPLIED WARRANTIES OF MERCHANTABILITY OR FITNESS FOR A PARTICULAR PURPOSE.

Acknowledgement

Funding for the RFC Editor function is currently provided by the Internet society. 